Original Research Paper

\title{
Wheeled Mobile Robot Trajectory Tracking using Sliding Mode Control
}

\author{
Azza El-Sayed Bayoumi Ibrahim \\ Department of Computers and Systems, Electronics Research Institute, Giza, Egypt
}

\section{Article history}

Received: 30-08-2015

Revised: $10-03-2016$

Accepted: 12-03-2016

Corresponding Author: Azza El-Sayed Bayoumi Ibrahim

Department of Computers and Systems, Electronics Research Institute, Giza, Egypt

Email: azza@eri.sci.eg

\begin{abstract}
This paper introduces and discusses a control strategy for nonholonomic wheeled mobile robots. The models of the robots include the kinematic and dynamic equations of motion. Trajectory tracking control problem of parallel wheeled differential drive mobile robot is considered, where the robot should reach the final position by following a referenced trajectory for different initial conditions. A motion control strategy for a mobile robot by only assuming the kinematic model was developed by many researchers. In the case of high-speed robot motion, the dynamical model is important. In this study, two stages of the proposed control strategy are presented. The first one is dealing with the kinematics of the system and denoted as 'steering' controller. The second one, a velocity controller is developed based on the robust sliding mode control technique. A new design of the sliding surface is proposed. The switching feedback gain is determined based on a novel mathematical simple rule, considering the initial state of the system. Robustness to parameters uncertainties and stability of the controlled system are achieved. A simulation model of the controlled system is developed in MATLAB-SIMULINK software. Simulation results show the performances of the developed controller. In the case of presence of uncertainties, the results show the superiority of the proposed controller compared with the computed torque method.
\end{abstract}

Keywords: Mobile Robot Kinematics and Dynamics, Sliding Mode Control, Trajectory-Tracking

\section{Introduction}

Mobile robots have been used in many applications and areas such as industrial, medical, etc. Wheeled Mobile Robots (WMRs) are considered as the most widely used class of mobile robots, due to their fast maneuvering and energy saving characteristics. In recent years, the issues of motion planning and control for WMRs have been widely pursued. The possible motion tasks can be classified as follows; Point-to-point motion, Path following motion and Trajectory tracking motion. In trajectory tracking problems, the robot must reach and follow a predefined trajectory in the Cartesian space, starting from a given initial position, on or off the trajectory (Laumond, 1998). Path planner or trajectory generation procedure should be done before the design of the tracking controller. Planning of continuous and smoothness curvature paths is done using several methods (Palamakumbura et al., 2013; Lin and Lian, 2005).
This paper concerns with trajectory tracking control motion. The majority of the publications in this area have focused on the control of only the kinematic vehicle models. Therefore, the control inputs of the mobile robot are often considered its linear and angular velocities as described in (Nițulescu, 2007; Saidonr et al., 2011). While less attention has been paid to the control problems of nonholonomic dynamic systems, where forces and torques are the true inputs. The dynamical model of WMR includes the total mass and the moment of inertia parameters. Thus, the designed controller deals with the dynamical effects which make it more applicable in the real world. Therefore, the dynamics of the vehicle should be considered and the feasible controllers should be implemented for both the kinematic and dynamic effects (Fierro and Lewis, 1997). There are different techniques can be used in controller design, one of the most common ones is the backsteeping method. This procedure is applied for solving control problems in the WMRs when the dynamic 
effects are taken into consideration (Zohar et al., 2011). The research papers of the robustness and control in the presence of the uncertainties in the dynamical model of such systems are sparse.

The proposed approach is based on the Sliding Mode Control technique (SMC). Sliding mode control is a special discontinuous control technique applicable to various practical systems (Andrzej and Zuk, 2010). SMC enjoys a wide variety of application areas, such as in robotics (Rezapour et al., 2014; Akhavan and Jamshidi, 2000) and in aerospace applications (Ibrahim et al., 2012), etc. The main advantages of using SMC are the fast response, relative simplicity of design, robustness on system uncertainties and external disturbances and good control performance for many nonlinear systems. Wang et al. (2007), two types of the sliding-mode controller for a class of under-actuated mechanical systems are developed. Qian et al. (2008), the hierarchical sliding mode control for the single-input system with saturation non-linearity is deduced by using Lyapunov theory.

This work concentrates on the accurate trajectory tracking control while optimal path planning is not within the scope of the paper. The capability of the underlying controller for solving the trajectory-tracking control problems is improved by using the control algorithm of (Ailon et al., 2005) which ensures in the case of the kinematic model exponential stability in tracking. Therefore, the feed forward kinematic controller and the feedback dynamic sliding mode controller are integrated ensuring the exponential convergence of the robot to the desired trajectory in a condition of the system uncertainties is bounded.

The paper is organized as follows. In section two, kinematic and dynamic state-space model of twowheeled mobile robots is introduced. Section three describes the problem has to be solved. Section four presents a well-known kinematic control law to compute the body linear and angular velocities required to control the robot following a planned trajectory. Section five details the design of a sliding mode controller to determine the required left and right torques that allow asymptotically perfect velocity tracking. The switching gain selection rule is discussed in section six. The seventh section shows simulation results to highlight the effectiveness of the proposed control scheme. In the last section, the SMC is compared with the computed torque method.

\section{Structure and Modeling of the Mobile Robot}

The considered mobile robot is composed of two similar driving wheels mounted on a bar and independently controlled by two actuators (motors), as indicated in Fig. 1. where $r$ is the wheel radius $(m), v_{L}$ and $v_{R}$ are left and right drive wheel velocities respectively $(\mathrm{m} / \mathrm{s}),(x, y)$ present mobile robot position point CG; center of axis of wheels in world Cartesian coordinates in $(\mathrm{m})$ and $2 L$ is axle length between the drive wheels $(m)$. The orientation of the mobile robot is given by the angle $\theta$ ( $\mathrm{rad}$ ) between the instant linear velocity of the mobile robot body, $v$ and the local vertical axis. The Position vector state of the robot is defined as $q$ $=(x, y, \theta)$. The linear and the angular velocities of the mobile robot are expressed by Equation 1 and the kinematic model equation of the mobile robot is given by Equation 2 (Laumond, 1998):

$v=\frac{v_{l}+v_{R}}{2}, \omega=\frac{v_{l}-v_{R}}{l}$

$\dot{q}=\left(\begin{array}{c}\dot{x} \\ \dot{y} \\ \dot{0}\end{array}\right)=\left(\begin{array}{cc}\cos \theta & 0 \\ \sin \theta & 0 \\ 0 & 1\end{array}\right)\left(\begin{array}{l}v \\ \omega\end{array}\right)$

The nonholonomic constraint is assumed, where the driving wheels roll purely and do not slip. Also, the dynamic model of a WMR is represented as in Equation 3, (Salem, 2013):

$\dot{y} \cos \theta-\dot{x} \sin \theta=0$
$M \dot{v}(t)-f_{f}(t)=F(t), \quad F(t)=\frac{\tau_{l}+\tau_{R}}{r}$
$I \dot{\omega}(t)-\tau_{f}(t)=\tau(t), \quad \tau(t)=\frac{l\left(\tau_{l}-\tau_{R}\right)}{r}$
let $u_{1}=\tau_{l}+\tau_{R}, \quad u_{2}=\tau_{l}-\tau_{R}$

Where:

$M \quad=$ The mass of entire mobile robot and

$I \quad=$ The moment of inertia of it

$\tau_{l}$ are $\tau_{R} \quad=$ The left-and right-wheel motor torques

$f_{f}(t)$ and $\tau_{f}(t)=$ The frictional force and frictional torque, respectively

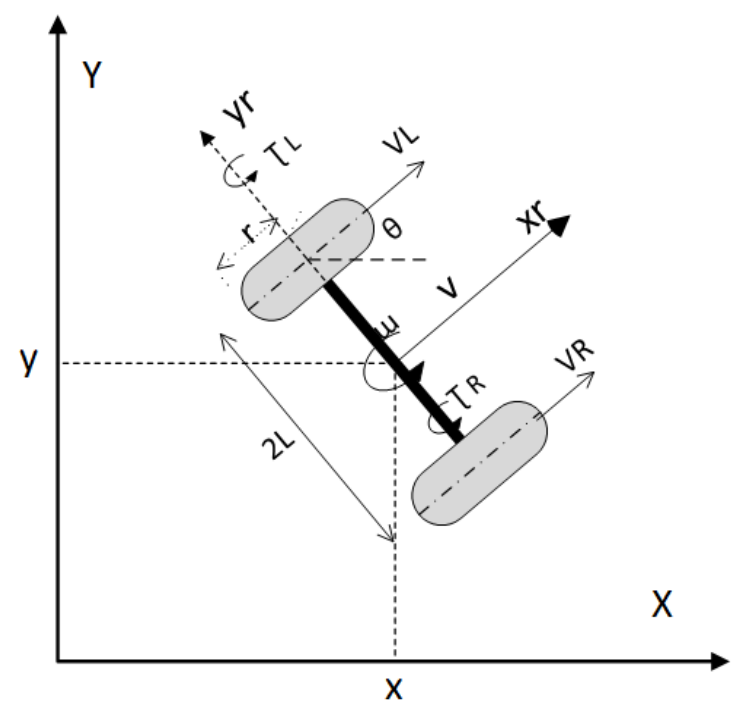

Fig. 1. A schematic model of the wheeled mobile robot 


\section{Problem Statement}

The subject of this paper is to control the WMR mobile robots to follow a predefined path moving on a plane, even when uncertainties exist. To clarify the problem, it can be assumed that a virtual vehicle is moving on the desired reference trajectory and the real robot must make typical motions as a virtual one as illustrated in Fig. 2. The virtual vehicle position and orientation trajectories and also its kinematic equations are described by Equation 4:

$$
\begin{aligned}
& q_{d}(t)=\left[x_{d}(t), y_{d}(t), \theta_{d}(t)\right]^{T} \\
& \dot{x}_{d}=v_{d} \cos \theta_{d}, \dot{y}_{d}=v_{d} \sin \theta_{d}, \dot{\theta}_{d}=\omega_{d}
\end{aligned}
$$

Besides, the desired reference time-varying linear and angular velocities can be computed as following:

$$
v_{d}=\sqrt{\dot{x}_{d}^{2}+\dot{y}_{d}^{2}} \text { and } \omega_{d}=\dot{\theta}_{d}=\frac{\ddot{y}_{d} \dot{x}_{d}-\ddot{x}_{d} \dot{y}_{d}}{\dot{x}_{d}^{2}+\dot{y}_{d}^{2}}
$$

Therefore, it is necessary to find the appropriate control law, such that $q(t) \rightarrow q_{q}$ as $t \rightarrow \infty$. The errors between the actual and desired positions are $e=\left[e_{1}, e_{2}, e_{3}\right]^{T}=\left[\left(x-x_{d}\right)\right.$, $\left.\left(y-y_{d}\right),\left(\theta-\theta_{d}\right)\right]^{T}$ Consequently, the error dynamics for trajectory tracking is described by Equation 6:

$$
\left[\begin{array}{c}
\dot{e}_{1} \\
\dot{e}_{2} \\
\dot{e}_{3}
\end{array}\right]=\left[\begin{array}{c}
v \cos \theta-v_{d} \cos d \\
v \sin \theta-v_{d} \sin d \\
\omega-\omega_{d}
\end{array}\right]
$$

The system controller is developed as two parts, a nonlinear kinematics (steering) controller whose outputs are linear and angular velocities (this is for kinematic stabilization). The second part is the dynamic controller whose outputs are the required linear and angular torques.

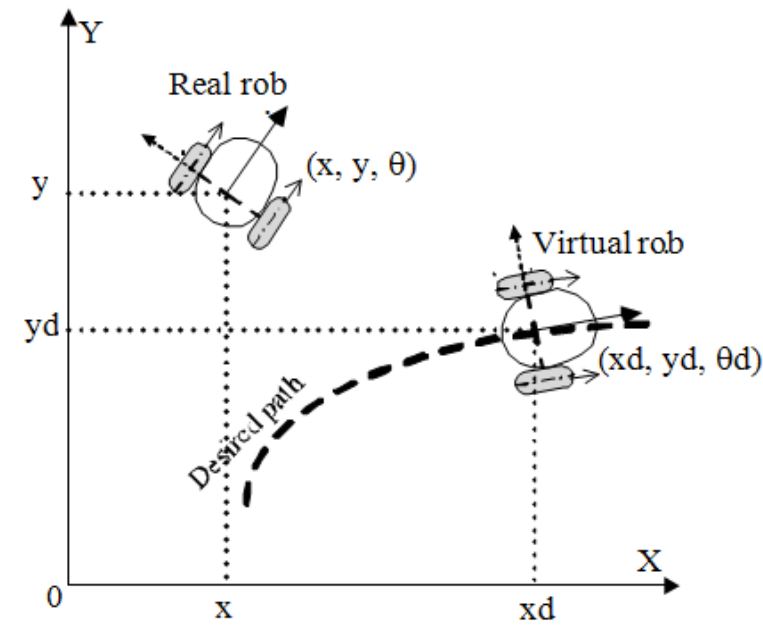

Fig. 2. The motion of real and virtual vehicles

\section{Kinematic Control Law Design}

Firstly, a control law for the kinematic model of the WMR is described by Equation 7, as described by (Zohar et al., 2011):

$v_{c}=\frac{v_{d} \cos \theta_{d}-\gamma e_{1}}{\cos \theta}, \omega_{c}=\omega_{d}-a e_{2}-b e_{3}$

where, a, b, $\gamma$ are positive gains constants. $v_{c}, \omega_{c}$ are the required linear and angular velocities for kinematic stabilization which are taken as reference inputs for the dynamic controller stage. Substituting Equation 7 into Equation 2 and using Equation 6 and elementary trigonometric identities to get the errors dynamics which are given by Equation 8:

$$
\begin{aligned}
& \dot{e}_{1}=-\gamma e_{1} \\
& \dot{e}_{2}=-\gamma e_{1} \tan \theta+\frac{v_{d} \sin e_{3}}{\cos \theta} \\
& \dot{e}_{3}=a e_{2}-b e_{3}
\end{aligned}
$$

By choosing the Lyapunov function described by Equation 9:

$V(e)=\frac{1}{2} e_{1}^{2}+\frac{1}{2} e_{2}^{2}+\frac{1}{2} e_{3}^{2}+e_{2} e_{3}$

It is proven that the mobile robot is able to asymptotically track the reference robots with the errors converge to zero as $t \rightarrow \infty$ (Ailon et al., 2005).

\section{Sliding Mode Controller Design}

The SMC method (Andrzej and Zuk, 2010) is utilized to design a dynamic tracking controller which let the actual velocities of the real robot converge to the control velocities generated from the kinematic controller. The completely controlled system block diagram is presented in Fig. 3. Firstly, the SMC is designed for the nominal system, i.e., the system parameters are known and disturbances are zero. The auxiliary velocities tracking errors and their derivatives are introduced as:

$$
\begin{aligned}
& v_{e}(t)=v(t)-v_{c}(t), \dot{v}_{e}(t)=\dot{v}(t)-\dot{v}_{c}(t) \\
& \omega_{e}(t)=\omega(t)-\omega_{c}(t), \dot{\omega}_{e}(t)=\dot{\omega}(t)-\dot{\omega}_{c}(t)
\end{aligned}
$$

The following integral-type sliding surface is proposed (Xu et al., 2014):

$$
\begin{aligned}
& s_{1}(t)=v_{e}(t)+c_{1} \int_{0}^{t} v_{e}(t) d t \\
& s_{2}(t)=\omega_{e}(t)+c_{2} \int_{0}^{t} \omega_{e}(t) d t
\end{aligned}
$$


where, $c_{1}$ and $c_{2}$ are strictly positive real constants determining the slope of the sliding surfaces. Based on SMC methodology, the control signal $u(t)$ is composed of two components as described by Equation 12:

$$
u(t)=u_{e q}(t)+u_{s w}(t)
$$

Based on the concept of the "equivalent" control, $u_{e q}$ is obtained by recognizing that $\dot{s}(t)$ is equal to zero, is a necessary condition for the state trajectory to stay on the sliding surface. The dynamic equations of mobile robot are reformulated, as $f_{f}(t)$ and $\tau_{f}(t)$ are equal to zeros and let $1 / M r, \beta=L / I r$ therefore:

$$
\dot{v}(t)=\frac{u_{1}(t)}{M r}=a u_{1}(t), \dot{\omega}(t)=\frac{L u_{2}(t)}{I r}=\beta u_{2}(t)
$$

Therefore, the equivalent control law is deduced as following:

$$
\dot{s}_{1}=\dot{v}_{e}+c_{1} v_{e}=0, \dot{s}_{2}=\dot{\omega}_{e}+c_{2} \omega_{e}=0
$$

Substituting into Equation 10 and 13 in Equation 14 then the derivative of $v_{c}, \omega_{c}$ is computed, therefore, the equivalent control signal components are determined as:

$$
\begin{aligned}
& u_{1 e q}=\left(\dot{v}_{c}-c_{1}\left(v-v_{c}\right)\right) / a \\
& u_{2 e q}=\left(\dot{\omega}_{d}-a \dot{e}_{2}-b \dot{e}_{3}-c_{2}\left(\omega-\omega_{c}\right)\right) / \beta
\end{aligned}
$$

The duty of the second component of the control signal, $u_{s w}$ of Equation 12 is to force the state trajectory to reach on sliding surface in finite time in spite of disturbances and model uncertainties in the system. For this target, the "constant rate" reaching law is chosen (Andrzej and Zuk, 2010):

$u_{i s w}=k_{i} \operatorname{sgn}\left(s_{i}\right), \quad i=1,2$

where, $k_{i}$ are positive constants, The switching gain $k$ should be chosen to compensate for the system uncertainties and disturbances. Thus, the total control law of the system is computed by Equation 17:

$$
\begin{aligned}
& u_{1}=\left(\dot{v}_{c}-c_{1}\left(v-v_{c}\right)-k_{1} \operatorname{sgn}\left(s_{1}\right)\right) / a \\
& u_{2}=\left(\dot{\omega}_{d}-a \dot{e}_{2}-b \dot{e}_{3}-c_{2}\left(\omega-\omega_{c}\right)-k_{2} \operatorname{sgn}\left(s_{2}\right)\right) / \beta
\end{aligned}
$$

In SMC, a fast oscillation about the sliding surface may be appeared resulting from the control signals. This undesirable oscillation is called the chattering phenomenon, (Bartoszewic and Aleksandra, 2009). To reduce the chattering phenomenon, the boundary layer method is the most common method. Thus replacing $s g n\left(s_{i}\right)$ in Equation 16 by the saturation function $s a t$ $\left(s_{i} / \in\right)$, where $\in$ is a small positive value which expresses the boundary layer thickness:

$\operatorname{sat}\left(s_{i}, \in_{i}\right)=\left\{\begin{array}{ll}1 & s_{i}>\epsilon_{i} \\ \frac{s}{\epsilon} & \left|s_{i}\right| \leq \epsilon_{i} \\ -1 & s_{i}<-\epsilon_{i}\end{array}\right\} i=1,2$

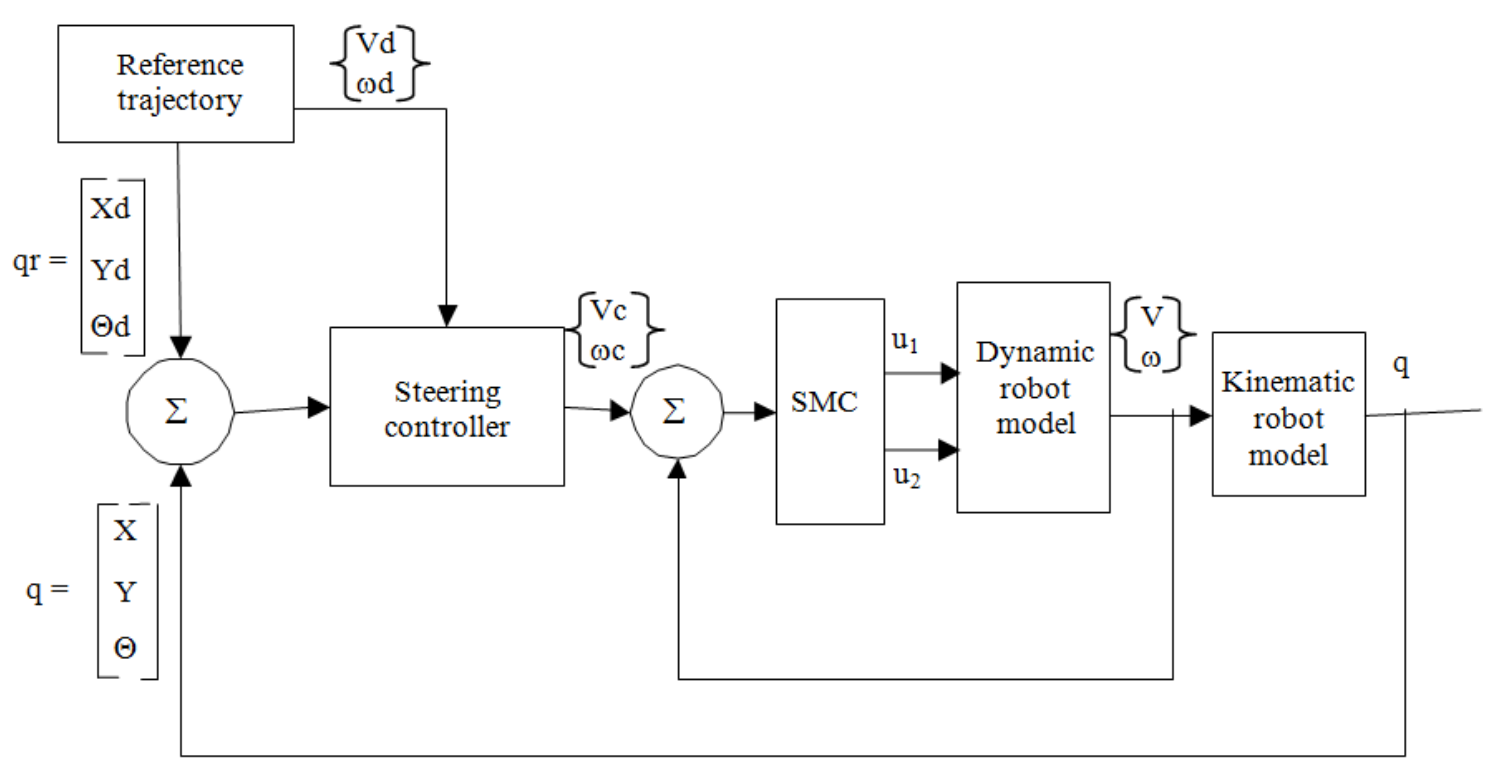

Fig. 3. Block diagram of the controlled system 


\section{The Switching Gain, $K_{i}$ Selection Rule}

The switching feedback gains $k_{i}$ can be automatically computed by a proposed simple rule. Based on the concept of the SMC technique, the switching gain is determined such that it satisfies the following reaching law, $s \dot{s} \leq-\eta|s|, \eta$ is a positive design scalar constant. Using Equation 14 in the selected reaching law:

$$
\begin{aligned}
& s\left(\dot{v}_{e}+c_{1} v_{e}\right) \leq-\eta|s| \\
& s\left(\dot{v}-\dot{v}_{c}+c_{1} v_{e}\right) \leq-\eta|s| \\
& s\left(a u_{1}+d+c_{1} v_{e}-\dot{v}_{c}\right) \leq-\eta|s|
\end{aligned}
$$

Substituting by $a u_{1}$ from Equation 17 into Equation 19 as follows:

$$
\begin{aligned}
& s\left(\dot{v}_{c}-c_{1}\left(v-v_{c}\right)-k_{1} \operatorname{sgn}\left(s_{1}\right)+d+c_{1} v_{e}-\dot{v}_{c}\right) \leq-\eta|s| \\
& s\left(-k_{1} \operatorname{sgn}\left(s_{1}\right)+d\right) \leq-\eta|s| \\
& k_{1} \geq\left|d_{1 \text { max }}\right|+\eta_{1}, \quad k_{2} \geq\left|d_{2 \max }\right|+\eta_{2}
\end{aligned}
$$

where, $\left|d_{\max }\right|$ is the upper bound of the disturbances which is assumed to be previously known. Therefore, $k i$ must be selected such that compensate for the upper bound of the disturbance.

In this study, from the definition of the variable, $s$ in Equation 11 it is noticed that the initial values of $S_{i}$ located on the vertical axis as shown in Fig. 4. The length of the perpendicular line (AN) from the initial state to the sliding surface "ds" is the shortest reaching distance. From the geometry of the figure, "ds" can be computed as following:

$$
|d s|=v_{e}(0) \sin \left(\frac{\pi}{2}-\tan ^{-1} c_{1}\right)
$$

According to Equation 20, the minimum values of $k_{1}$, $k_{2}$ are deduced as in Equation 22:

$$
k_{1}=\left|d_{1 \text { max }}\right|+\left|d s_{1}\right|, k_{2}=\left|d_{2 \max }\right|+\left|d s_{2}\right|
$$

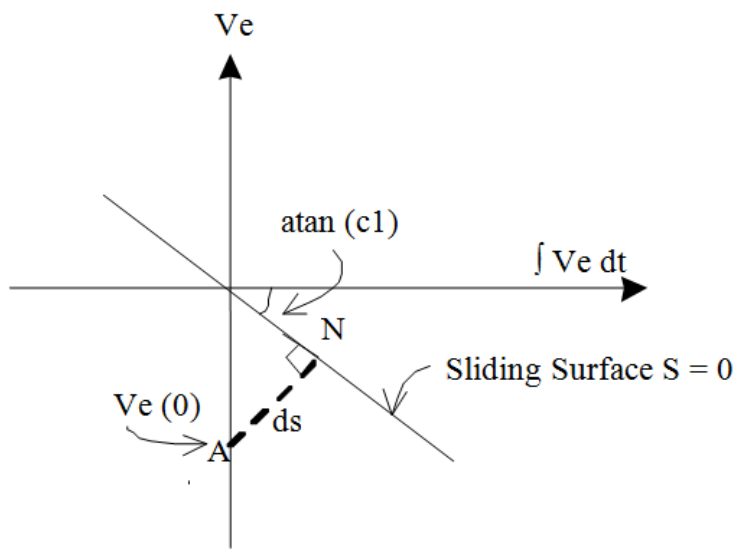

Fig. 4. Feedback gain selection rule
The stability of the SMC can be verified by choosing a non-negative quadratic function $V=\frac{1}{2} s_{1}^{2}+\frac{1}{2} s_{2}^{2}$ as Lyapunov function. The derivative of it is $\dot{V}=s_{1} \dot{s}_{1}+s_{2} \dot{S}_{2}$. Using Equation 11 and 14, it is found that, $\dot{V} \leq 0$, therefore, $s$ reaches zero. So the system is stable. This ensures the stability of the dynamic control loop.

\section{Simulation Results}

To demonstrate the effectiveness of the proposed control scheme, the complete system is simulated using MATLAB/Simulink software. The desired geometric path is given by $y=g(x)=\sin (0.5 x)+0.5 x+1$ and the projection of the trajectory along the $x$-axis is $x(t)=t$. Model input is also built to generate the desired linear and angular velocities of the virtual mobile robot as shown in Fig. 3. The parameters of the robot in Fig. 1 are assumed to be known. The robot mass is $m=9 \mathrm{~kg}$, the wheel radius $r=1 \mathrm{~m}$ and the total inertia is $5 \mathrm{kgm}^{2}$.

In the beginning, the simulations with no friction or uncertainty are executed for tracking the defined trajectory.

For a case study, it is assumed that the actual mobile robot starts from the initial velocities $v(0)=0$ and $\omega$ (0)-1 and the initial position $q(0)=[3,2,0]^{T}$, (the desired path start point is $(0,1,0.78))$.

Several experiments for different values of the gains have done. Figure 5 and 6 show the responses of the trajectory tracking control of the WMR when there are no perturbations and there is a large initial posture error. Figure $5 \mathrm{a}$ and $5 \mathrm{~b}$ declare the behaviours of the robot along the $\mathrm{x}$-direction and $\mathrm{y}$-direction. Figure $5 \mathrm{c}$ describes the head angle (the orientation) behaviour of the robot. It is clear that the actual vehicle successively follows the virtual one (desired trajectory) after $10 \mathrm{sec}$. Figure 6 shows the decaying behavior of the errors of the linear and angular velocities. The initial states and the results are recorded and the designed parameters of the proposed controller are also defined in Table 1. The system responses proved that the proposed controller possesses good tracking ability and very good response. The boundary layer thickness to eliminate the chattering is selected as $\epsilon_{1}=0.01, \epsilon_{2} 0.05$.

Another test case is simulated to verify the robustness of the proposed controller; a bounded uncertainty is applied. The robot mass and its inertia are changed with $10 \%$ of its nominal values. The planned path and the initial conditions of the robot are the same as those of the previous case.

The results indicate that uncertainties will slightly degrade the response, a very small increasing in tracking error occurs such that at steady state the errors become $\left(e_{s . s 1}=-2.122 \mathrm{e}-5, e_{s . s 2}=-0.000184, e_{s . s 3}=-0.000141, v_{e_{s . s}}\right.$ $\left.=-0.0002352, \omega_{\text {es.s }},=-0.00118\right)$. 


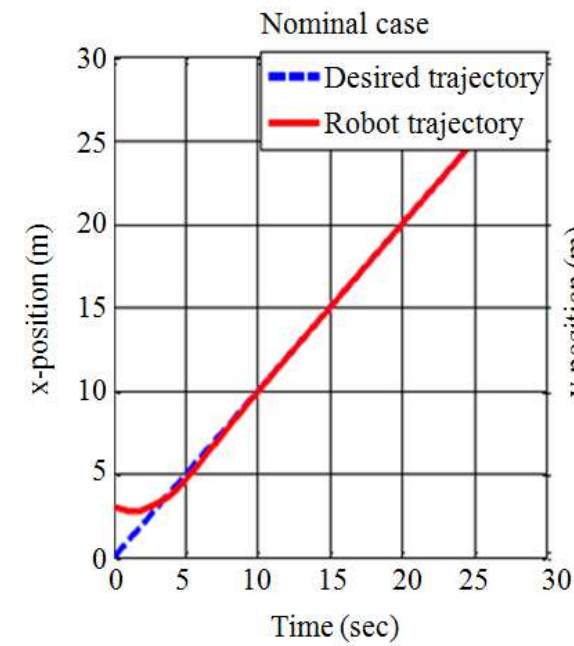

(a)

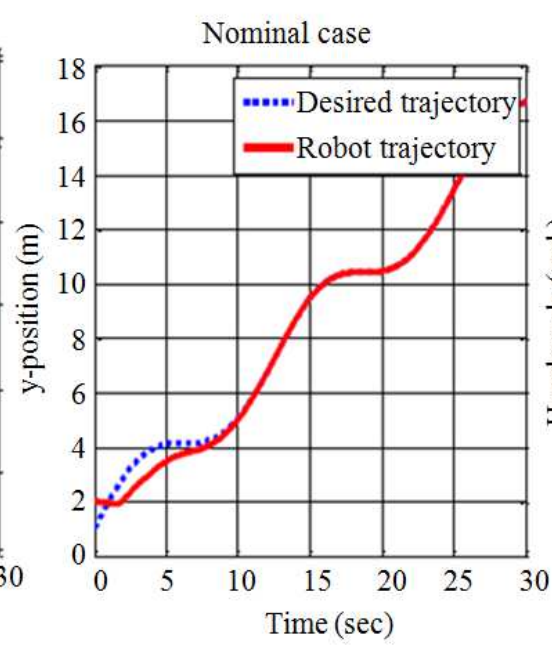

(b)

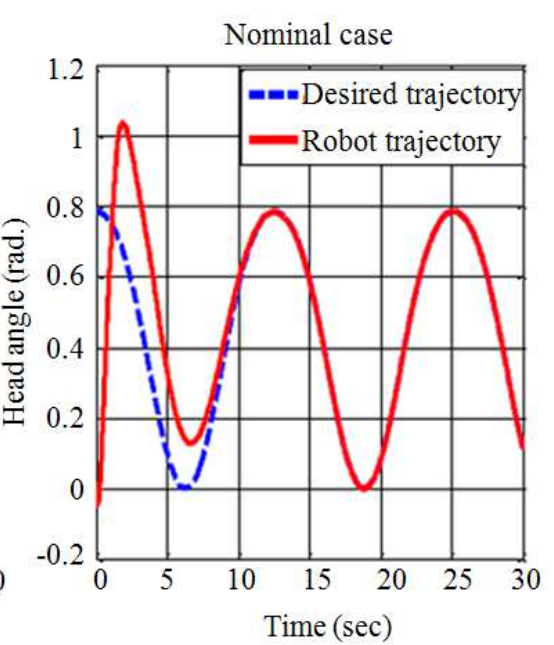

(c)

Fig. 5. The system responses: (a) in x-direction, (b) in y-direction and (c) the robot head angle

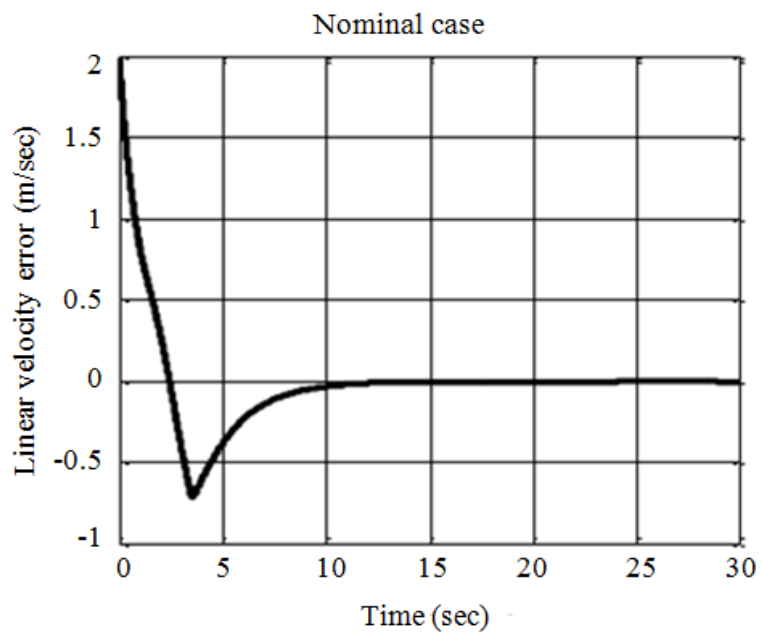

(a)

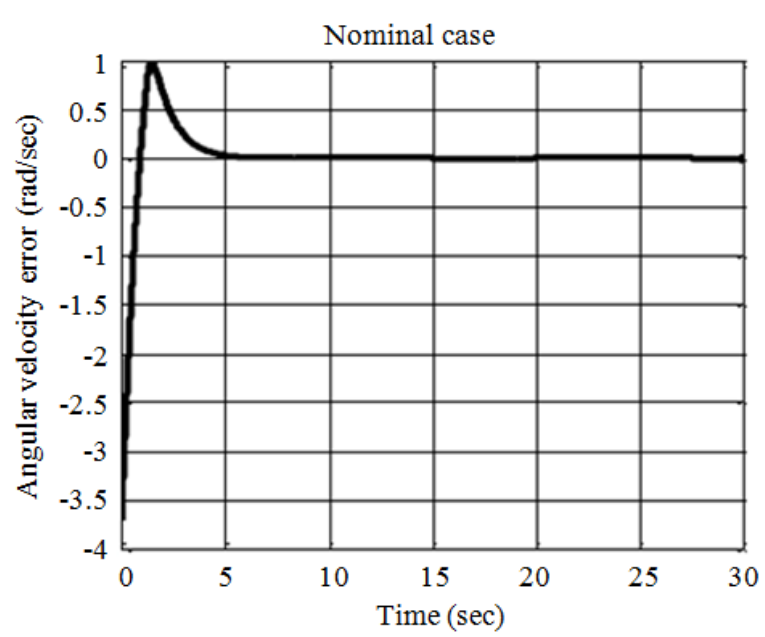

(b)

Fig. 6. (a) Tracking error of forward velocity $\left(v-v_{c}\right)$, (b) Tracking error of angular velocity $\left(\omega-\omega_{c}\right)$

Table 1. The controller parameters and the results in case of free uncertainties (nominal) system

\begin{tabular}{|c|c|c|c|c|c|c|c|}
\hline Initial states & $\mathrm{X}(0)$ & $\mathrm{Y}(0)$ & $\theta(0)$ & $\mathrm{V}(0)$ & & $\omega(0)$ & \\
\hline Actual robot & 3 & 2 & 0 & 0 & & -1 & \\
\hline Ref. trajectory & 0 & 1 & 0.785 & 1.414 & & 0 & \\
\hline Controller parameters & $\gamma$ & a & $\mathrm{b}$ & $\mathrm{K} 1$ & $\mathrm{~K} 2$ & $\mathrm{C} 1$ & $\mathrm{C} 2$ \\
\hline & 1 & 2 & 6 & 1.79 & 2.63 & 0.5 & 1 \\
\hline Steady-state errors & $\begin{array}{l}\mathrm{e}_{1}(\mathrm{~s} . \mathrm{s}) \\
-3.135 \mathrm{e}-6\end{array}$ & $\begin{array}{l}\mathrm{e}_{2}(\mathrm{~s} . \mathrm{s}) \\
-0.000188\end{array}$ & $\begin{array}{l}\mathrm{e}_{3}(\mathrm{~s} . \mathrm{s}) \\
0.000147\end{array}$ & $\begin{array}{l}v_{\mathrm{c}}(\mathrm{s} . \mathrm{s}) \\
-1.47 \mathrm{e}-6\end{array}$ & & $\begin{array}{l}\omega_{\mathrm{e}}(\mathrm{s} . \mathrm{S}) \\
-0.00122\end{array}$ & \\
\hline
\end{tabular}

In this case, the higher switching gains are needed and selected equal $\left(k_{1}=1.949, k_{2}=2.848\right)$. However, the higher controller gains are giving rise to large control efforts and high chattering. Consequently, the larger boundary layer thickness is needed to eliminate appearing of chattering phenomena in the control signal. The selected values of thickness are equal to $\left(\epsilon_{1}=0.1, \epsilon_{2}=0.5\right)$.

Finally, as we can see from the experimental results, the trajectory tracking is very robust to the parameter uncertainties using the proposed method. 


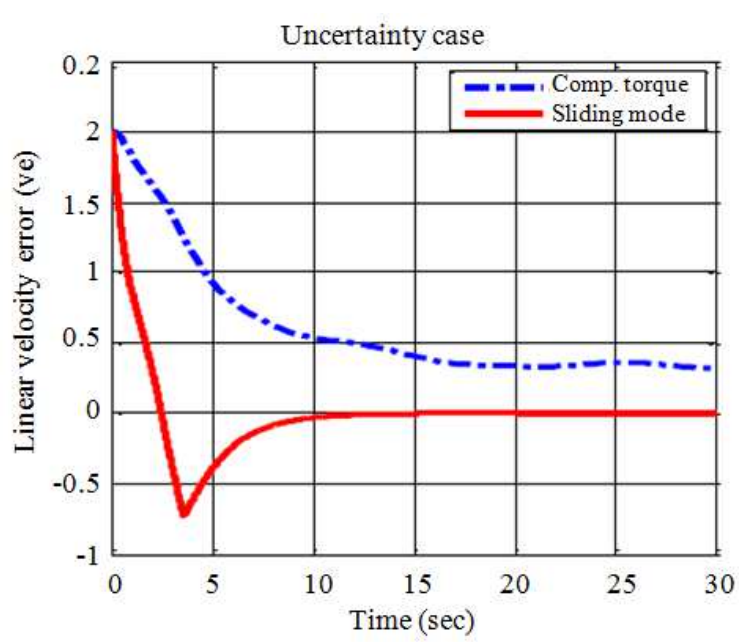

(a)

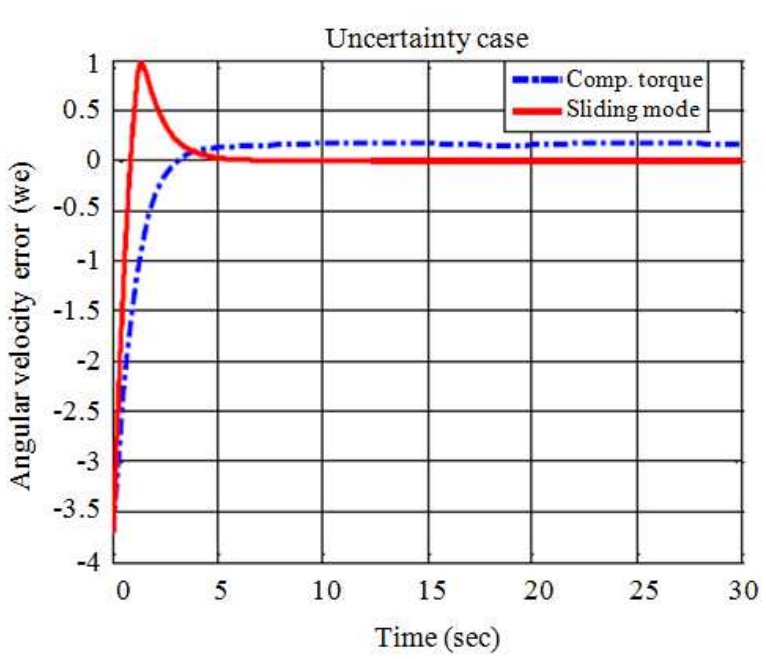

(b)

Fig. 7. (a) The linear velocity error signals of computed torque method and SMC, (b) The angular velocity error signals of computed torque method and SMC

\section{Comparative Study}

In this section, the proposed control law is compared with the Computed Torque Control method (CTC). CTC method is used in the dynamical control loop instead of the SMC method as in the system block diagram of Fig. 3. The control torque signals are computed according to Equation 23 as described in (Divya, 2012):

$$
\begin{aligned}
& a u_{1}=v_{\dot{c}}+\frac{k_{l}}{M}\left(v_{c}-v\right) \\
& \beta u_{2}=\omega_{\dot{c}}+\frac{k_{a}}{1}\left(\omega_{c}-\omega\right)
\end{aligned}
$$

where, $k_{l}$ and $k_{a}$ are two positive control parameters. Simulation studies have been performed to test both controllers using the same conditions and parameters uncertainties. Figure $7 \mathrm{a}$ and $7 \mathrm{~b}$ clearly show the performance of the SMC compared with the computed torque controller. As shown in figures, the SMC controller follows the reference trajectory with a very small or negligible steady state errors, but the computed torque controller shows high steady state error in case of existing of uncertainties in the total mass and inertia parameters with $10 \%$ of its nominal values. Therefore, the sliding mode controller is more robust. In addition, the selection of the controller gains in computed torque method must be recalculated for any change in the system parameters. It is clearly from Fig. 7 that the steady state errors are: $v_{e}$ s.s $=0.3175$ and $\omega_{e} s . s=0.1623$ by CTC and the SMC steady state errors are $v_{e} s . s=-0.000218$ and $\omega_{e} s . s=-0.00112$.

\section{Conclusion}

This paper presents control schemes for WMRs whose models include the kinematic and the dynamic models. The nonlinear modeling of the mobile robot is considered. A new approach for solving the trajectorytracking problem of a nonholonomic mobile robot is proposed. A nonlinear model of the robot is controlled using integral sliding mode control method to achieve the target position along the predefined desired path. A simple mathematical rule is suggested to select the switching feedback gains of the SMC for different initial conditions.

The robot using the suggested approach is successfully tracking the reference desired trajectory. From the obtained results, the sliding mode control technique is effective controller because the error is decaying to zero.

A comparison study of the sliding mode controller and the computed torque method is done. The simulation results show that the robot under the proposed controller tracks the desired trajectory with very tiny steady state errors in case of the presence of parametric uncertainties. Therefore, the sliding mode controller is more accurate than the computed torque method.

Finally, the obtained results show the efficiency, the simplicity and the accuracy of the proposed control strategy.

\section{Acknowledgement}

The author wish to thank reviewers for their valuable, comments that improve both the content and representation of this article. 


\section{Funding Information}

The authors have no support or funding to report.

\section{Ethics}

This article is original and contains unpublished material. The corresponding author confirms that all of the other authors have read and approved the manuscript and no ethical issues involved.

\section{References}

Ailon, A., N. Berman and S. Arogeti, 2005. On controllability and trajectory tracking of a kinematic vehicle model. Automatica, 41: 889-896. DOI: $10.1016 /$ j.automatica.2004.11.025

Akhavan, S. and M. Jamshidi, 2000. ANN-based sliding mode control for non-holonomic mobile robots. Proceedings of the IEEE International Conference on Control Applications Anchorage, (CAA' 00), Alaska, USA September pp: 25-27. DOI: $10.1109 /$ CCA.2000.897506

Andrzej, B. and J. Zuk, 2010. Sliding mode control-basic concepts and current trends. Proceedings of the International Symposium on Industrial Electronics, Jul. 4-7, IEEE Xplore Press, Bari, pp: 3772-3777. DOI: 10.1109/ISIE.2010.5637990

Bartoszewic, A. and N.L. Aleksandra, 2009. TimeVarying Sliding Modes for Second and Third Order Systems. 1st Edn., Springer Science and Business Media, Berlin, ISBN-10: 3540922164, pp: 192.

Divya, A., 2012. Tracking controller of mobile robot. Proceedings of the International Conference on Computing, Electronics and Electrical Technologies, (EET' 12) IEEE Xplore Press, Kumaracoil, pp: 343-349.

DOI: 10.1109/ICCEET.2012.6203785

Fierro, R. and F.L. Lewis, 1997. Control of a nonholonomic mobile robot: Backstepping kinematics into dynamics. J. Robotic Syst., 14: 149-163.

Ibrahim, A., M. Ahamed and A. Mohammad, 2012. Satellite attitude maneuver using sliding mode control under body angular velocity constraints. Int. J. Comput. Applic., 50: 0975-8887.

DOI: $10.5120 / 7835-1133$

Laumond, J.P., 1998. Robot Motion Planning and Control. 1st Edn., Springer, London, ISBN-10: 3540762191, pp: 343.

Lin, S.H. and F.L. Lian, 2005. Study of feasible trajectory generation algorithms for control of planar mobile robots. Proceedings of the IEEE International Conference on Robotics and Biomimetics, (CRB' 05), IEEE Xplore Press, Shatin, pp: 121-126.

DOI: 10.1109/ROBIO.2005.246249
Niţulescu, M., 2007. Solutions for modeling and control in mobile robotics. CEAI, 9: 43-50.

Palamakumbura, R., D.H.S. Maithripala and C.F. Martin, 2013. Minimum jerk trajectory generation for differential wheeled mobile robots. Proceedings of the 8th International Conference on Industrial and Information Systems, Dec.17-20, IEEE Xplore Press, Peradeniya, pp: 460-464. DOI: $10.1109 /$ ICIInfS.2013.6732028

Qian, D., J. Yi and D. Zhao, 2008. Control of a class of under-actuated systems with saturation using hierarchical sliding mode. Proceedings of the International Conference on Robotics and Automation, May 19-23, IEEE Xplore Press, Pasadena, CA, pp: 2429-2434.

DOI: 10.1109/ROBOT.2008.4543577

Rezapour, E., K.Y. Pettersen, P. Liljebäck and J.T. Gravdahl, 2014. Differential geometric modelling and robust path following control of snake robots using sliding mode techniques. Proceedings of the IEEE International Conference on Robotics and Automation, May 31-Jun. 7, IEEE Xplore Press, Hong Kong, pp: 4532-4539.

DOI: 10.1109/ICRA.2014.6907521

Saidonr, M.S., H. Desa and M.N. Rudzuan, 2011. A differential steering control with proportional controller for an autonomous mobile robot. Proceedings of the 7th International Colloquium on Signal Processing and its Applications, Mar. 4-6, IEEE Xplore Press, Penang, pp: 90-94. DOI: $10.1109 /$ CSPA.2011.5759849

Salem, F.A., 2013. Kinematics and dynamic models and control for differential drive mobile robots. Int. J. Current Eng. Technol., 3: 253-263.

Wang, W., X.D. Liu and J. Yi, 2007. Structure design of two types of sliding-mode controllers for a class of under-actuated mechanical systems. IET Control Theory Appllic., 1: 163-172. DOI: 10.1049/iet-cta:20050435

Xu, J., Z. Guo and T.H. Lee, 2014. Design and implementation of integral sliding-mode control on an underactuated two-wheeled mobile robot. IEEE Trans. Indust. Electron., 61: 3671-368. DOI: 10.1109/TIE.2013.2282594

Zohar, I., A. Ailon and R. Rabinovici, 2011. Mobile robot characterized by dynamic and kinematic equations and actuator dynamics: Trajectory tracking and related application. Robotics Autonomous Syst., 59: 343-353. DOI: 10.1016/j.robot.2010.12.001 